\title{
Telomerase Reverse Transcriptase Promoter Mutations in Bladder Cancer: High Frequency Across Stages, Detection in Urine, and Lack of Association with Outcome
}

\author{
Yves Allory $^{a, b, 1}$, Willemien Beukers ${ }^{c, 1}$, Ana Sagrera $^{a}$, Marta Flández $^{a}$, Miriam Marqués ${ }^{a}$, \\ Mirari Márquez ${ }^{d}$, Kirstin A. van der Keur ${ }^{c}$, Lars Dyrskjot ${ }^{e}$, Irene Lurkin ${ }^{c}$, Marcel Vermeij $^{c}$, \\ Alfredo Carrato ${ }^{f}$, Josep Lloreta $^{g, h}$, José A. Lorente $^{i}$, Enrique Carrillo-de Santa Pau ${ }^{a}$, \\ Roy G. Masius ${ }^{c}$, Manolis Kogevinas ${ }^{j, k, l, m}$, Ewout W. Steyerberg ${ }^{n}$, Angela A.G. van Tilborg ${ }^{c}$, \\ Cheno Abas $^{c, a}$, Torben F. Orntoft ${ }^{e}$, Tahlita C.M. Zuiverloon ${ }^{c}$, Núria Malats ${ }^{d}$,
} Ellen C. Zwarthoff ${ }^{c, 2}$, Francisco X. Real ${ }^{a, h, 2, *}$

\begin{abstract}
${ }^{a}$ Epithelial Carcinogenesis Group, Molecular Pathology Program, CNIO (Spanish National Cancer Research Center), Madrid, Spain; ${ }^{\mathrm{b}}$ Université Paris-Est Créteil, Institut Mondor de Recherche Biomédicale, Créteil, France; ${ }^{\mathrm{c}}$ Department of Pathology, Erasmus MC, Rotterdam, The Netherlands; ${ }^{\mathrm{d}}$ Genetic and Molecular Epidemiology Group, Human Cancer Genetics Program, CNIO (Spanish National Cancer Research Center), Madrid, Spain; ${ }^{\mathrm{e} D e p a r t m e n t ~ o f ~}$ Molecular Medicine, Aarhus University Hospital, Aarhus, Denmark; ${ }^{\mathrm{f}}$ Medical Oncology Department, Ramón y Cajal University Hospital, Madrid, Spain; ${ }^{\mathrm{g}}$ Department of Pathology, Hospital del Mar-Parc de Salut Mar, Barcelona, Spain; ${ }^{\mathrm{h}}$ Departament de Ciències Experimentals i de la Salut, Universitat Pompeu Fabra, Barcelona, Spain; ${ }^{\mathrm{i}}$ Urology Service, Hospital del Mar-Parc de Salut Mar, Barcelona, Spain; ${ }^{\mathrm{j}}$ Centre de Recerca d'Epidemiologia Ambiental, Barcelona, Spain; ${ }^{\mathrm{k}}$ IMIM-Institut de Recerca Hospital del Mar, Barcelona, Spain; ${ }^{1}$ CIBER Epidemiología y Salud Pública (CIBERESP), Barcelona, Spain; ${ }^{\mathrm{m}}$ National School of Public Health, Athens, Greece; ${ }^{\mathrm{n}}$ Department of Public Health, Erasmus MC, Rotterdam, The Netherlands
\end{abstract}

\section{Article info}

Article history:

Accepted July 30, 2013

Published online ahead of

print on September 7, 2013

\section{Keywords:}

Bladder cancer

TERT gene

Somatic mutations

Clinical end point

Urine-based diagnosis

\begin{abstract}
Background: Hotspot mutations in the promoter of the gene coding for telomerase reverse transcriptase (TERT) have been described and proposed to activate gene expression.

Objectives: To investigate TERT mutation frequency, spectrum, association with expression and clinical outcome, and potential for detection of recurrences in urine in patients with urothelial bladder cancer (UBC).

Design, setting, and participants: A set of 111 UBCs of different stages was used to assess TERT promoter mutations by Sanger sequencing and TERT messenger RNA (mRNA) expression by reverse transcription-quantitative polymerase chain reaction. The two most frequent mutations were investigated, using a SNaPshot assay, in an independent set of 184 non-muscle-invasive and 173 muscle-invasive UBC (median follow-up: $53 \mathrm{mo}$ and $21 \mathrm{mo}$, respectively). Voided urine from patients with suspicion of incident UBC $(n=174)$, or under surveillance after diagnosis of non-muscle-invasive UBC $(n=194)$, was tested using a SNaPshot assay.

Outcome measurements and statistical analysis: Association of mutation status with age, sex, tobacco, stage, grade, fibroblast growth factor receptor 3 (FGFR3) mutation, progression-free survival, disease-specific survival, and overall survival.

1 Authors contributed equally.

2 Authors contributed equally.

* Corresponding author. Epithelial Carcinogenesis Group, Molecular Pathology Program, Centro Nacional de Investigaciones Oncológicas, Melchor Fernández Almagro, 3, 28029 Madrid, Spain. Tel. +34 917328000 ext 3660

E-mail address: preal@cnio.es (F.X. Real).
\end{abstract}


Results and limitations: In the two series, 78 of 111 (70\%) and 283 of 357 (79\%) tumors harbored TERT mutations, C228T being the most frequent substitution (83\% for both series). TERT mutations were not associated with clinical or pathologic parameters, but were more frequent among FGFR3 mutant tumors $(p=0.0002)$. There was no association between TERT mutations and mRNA expression $(p=0.3)$. Mutations were not associated with clinical outcome. In urine, TERT mutations had $90 \%$ specificity in subjects with hematuria but no bladder tumor, and $73 \%$ in recurrence-free UBC patients. The sensitivity was $62 \%$ in incident and $42 \%$ in recurrent UBC. A limitation of the study is its retrospective nature.

Conclusions: Somatic TERT promoter mutations are an early, highly prevalent genetic event in UBC and are not associated with TERT mRNA levels or disease outcomes. A SNaPshot assay in urine may help to detect UBC recurrences.

(C) 2013 European Association of Urology. Published by Elsevier B.V. All rights reserved.

\section{Introduction}

Urothelial bladder cancer (UBC) is heterogeneous at the clinical, pathologic, and genetic levels. Approximately $75 \%$ of newly diagnosed tumors are non-muscle invasive (Ta, Tis, and T1); most of them recur and $15-20 \%$ progress to invade muscle. The remaining $25 \%$ of patients present with muscle-invasive tumors and have a 5-yr survival of $<50 \%$ [1-3]. Fibroblast growth factor receptor 3 (FGFR3) is the most commonly mutated gene in UBC, with an overall frequency of 60\% [4-6]. Mutations in Harvey rat sarcoma viral oncogene homolog (HRAS), neuroblastoma RAS viral (v-ras) oncogene homolog (NRAS), Kirsten rat sarcoma viral oncogene homolog (KRAS), and phosphatidylinositol-4,5bisphosphate 3-kinase, catalytic subunit alpha (PIK3CA) are less frequent [6,7]. FGFR3 mutations are associated with stage and grade and are very common in pTa and grade $1 / 2$ tumors. This makes FGFR3 mutation analysis in voided urine a useful tool for diagnosing recurrent disease after a primary non-muscle-invasive bladder cancer (NMIBC) $[8,9]$.

Telomerase increases telomere length at chromosome ends. This activity is crucial for proliferating cells: Without telomerase, chromosomes are shortened every cell-division cycle, leading to replicative senescence and genomic instability $[10,11]$. Telomerase is active in stem cells but becomes downregulated in differentiated cells in somatic tissues. By contrast, it becomes reactivated in many tumors. Somatic hotspot mutations in the promoter of the gene coding for telomerase reverse transcriptase (TERT) catalytic subunit have recently been described in $71 \%$ of sporadic melanomas; germline mutations have also been reported in a melanoma kindred $[12,13]$. The somatic mutations were mutually exclusive C-to- $\mathrm{T}$ transitions at nucleotides $1,295,228$ (C228T) and 1,295,250 (C250T). These are the first hotspot somatic mutations reported in a gene promoter in human cancers and have been proposed to enhance TERT expression through the creation of novel binding sites for E-twenty-six (ETS)/ELK transcription factors [12,13]. Nevertheless, direct proof for this mechanism is absent. Interestingly, Huang et al. reported the C228T mutation in three of three UBC cell lines [12] and, in a survey of different tumor types, TERT promoter mutations were also found in UBC, hepatocarcinoma, glioblastoma, oligodendroglioma, and myxoid liposarcomas, but not in breast, colorectal, and prostate cancers [14]. However, the number of bladder tumors analyzed was small and clinical or pathologic information was not available [14].

In this paper, we investigate the frequency of TERT promoter mutations in two large series of UBC. In the first one, lacking outcome information, we assessed the relationship between TERT mutations and TERT messenger RNA (mRNA) expression; in the second, with patient follow-up, we assessed the relationship between mutations and outcome. Finally, we explored whether mutation detection in urine may be used to identify tumor recurrence.

\section{Materials and methods}

\subsection{Patient characteristics}

A set of 111 patients with associated frozen tumor samples came from the Spanish Bladder Cancer/EPICURO Study $[15,16]$ and from the Integrated Study of Bladder Cancer (ISBLAC). Clinical and sociodemographic information was retrieved from hospital records through a structured questionnaire. Subjects were defined as former smokers if they quit smoking $\geq 1 \mathrm{yr}$ before the date of interview. A second set of 357 patients, with associated formalin-fixed paraffin embedded (FFPE) tumor samples and follow-up information, came from the Erasmus Medical Center and other hospitals in the Netherlands. This cohort comprises 184 patients with primary NMIBC selected to contain a 50:50 distribution of cases with or without progression; the latter was defined by the development of MIBC. It also comprises 173 patients who have undergone a cystectomy for primary MIBC, stage pT2-4. Cause of death for patients in the MIBC cohort was determined from hospital records and/or direct contact with general practitioners. Staging and grading were performed according to the TNM 2002 classification and the three-grade 1973 World Health Organization classification. To confirm staging/grading and ensure uniformity of classification criteria, expert pathologists reviewed diagnostic slides from all tumor blocks. Table 1 shows the characteristics of patients whose tumor samples were studied.

For the urine study, samples were obtained from a consecutive, prospectively sampled collection of urine specimens from two centers (Hospital del Mar, Barcelona, Spain; and Erasmus MC, Rotterdam, Netherlands) participating in the European Community Seventh Framework program-funded "Prediction of bladder cancer disease course using risk scores that combine molecular and clinical risk factors" (UROMOL) study (EU-7FP UROMOL \#201663). Samples were obtained prior to cystoscopy at the time when a primary UBC was diagnosed $(n=135)$, or from patients lacking a prior history of UBC who presented with hematuria for diagnostic work-up and lacked visible bladder tumors at cystoscopy $(n=39)$. Additional samples $(n=395)$ were obtained during surveillance for relapsed disease (recurrence or progression) in 194 patients with NMIBC. Primary tumor or recurrence was defined by the presence of a 
Table 1 - Characteristics of patients whose tumor samples were studied

\begin{tabular}{|c|c|c|c|c|}
\hline Characteristic & Variable & $\begin{array}{l}\text { Spain } \\
(n=111), \\
\text { no. }(\%)\end{array}$ & $\begin{array}{l}\text { The Netherlands, NMIBC } \\
\qquad \begin{array}{c}(n=184), \\
\text { no. }(\%)\end{array}\end{array}$ & $\begin{array}{c}\text { The Netherlands } \\
\text { MIBC } \\
(n=173), \\
\text { no. (\%) }\end{array}$ \\
\hline Age, yr, median & & 76 & 70 & 71 \\
\hline \multirow[t]{2}{*}{ Sex } & Female & $13(12)$ & $46(25)$ & $28(16)$ \\
\hline & Male & $98(88)$ & $138(75)$ & $145(84)$ \\
\hline \multirow[t]{5}{*}{ Stage } & $\mathrm{pTa}+\mathrm{pTis}$ & $63(57)$ & $107(58)$ & - \\
\hline & pT1 & $28(25)$ & $77(42)$ & - \\
\hline & pT2 & $13(12)$ & - & $72(42)$ \\
\hline & pT3 & $3(3)$ & - & $75(43)$ \\
\hline & pT4 & $3(3)$ & - & $26(15)$ \\
\hline \multirow[t]{3}{*}{ Grade } & G1 & $27(24)$ & $14(8)$ & $1(1)$ \\
\hline & G2 & $28(25)$ & $101(55)$ & $13(7.5)$ \\
\hline & G3 & $56(51)$ & $69(37)$ & $159(92)$ \\
\hline Follow-up, mo, median & & - & 53 & 21 \\
\hline \multirow[t]{6}{*}{ End point } & Progression to MIBC & - & $90(49)$ & - \\
\hline & Local or distant progression to MIBC & - & - & $78(45)$ \\
\hline & Died of disease & - & - & $65(37)$ \\
\hline & Died of other causes & - & - & $36(21)$ \\
\hline & Alive at follow-up & - & - & $37(21)$ \\
\hline & Lost to follow-up & - & - & $35(20)$ \\
\hline \multirow[t]{2}{*}{ TERT status } & Mut & $78(70)$ & $147(80)$ & $136(79)$ \\
\hline & WT & $33(30)$ & $37(20)$ & $37(21)$ \\
\hline \multirow{4}{*}{ TERT mutations" } & $\mathrm{C} 228 \mathrm{~T}$ & $65(83)$ & $121(82)$ & $113(83)$ \\
\hline & $\mathrm{C} 228 \mathrm{~A}$ & $1(1.5)$ & $3(2)$ & $3(2)$ \\
\hline & CC242/243TT & $2(2.5)$ & NA & NA \\
\hline & $\mathrm{C} 250 \mathrm{~T}$ & 10 (13) & $23(16)$ & $20(15)$ \\
\hline
\end{tabular}

Table 2 - Characteristics of tumors and patients used for urine diagnostic assays

\begin{tabular}{|c|c|c|c|}
\hline Characteristic & Variable & $\begin{array}{l}\text { The Netherlands } \\
\text { no. (\%) }\end{array}$ & $\begin{array}{l}\text { Spain } \\
\text { no. (\%) }\end{array}$ \\
\hline $\begin{array}{l}\text { Patients with primary } \\
\text { tumor diagnosis }\end{array}$ & Patients, no. & 45 & 90 \\
\hline \multirow[t]{4}{*}{ Stage } & pTa & $39(87)$ & $53(59)$ \\
\hline & pTis & & $3(3)$ \\
\hline & pT1 & $5(11)$ & $21(23)$ \\
\hline & pT2-4 & $1(2)$ & $13(15)$ \\
\hline \multirow[t]{3}{*}{ Grade } & G1 & $17(38)$ & $27(30)$ \\
\hline & G2 & $26(58)$ & 17 (19) \\
\hline & G3 & $2(4)$ & $46(51)$ \\
\hline $\begin{array}{l}\text { Patients under surveillance } \\
\text { after NMIBC diagnosis" }\end{array}$ & Patients, no. & 118 & 76 \\
\hline \multicolumn{4}{|l|}{ With relapsing tumors } \\
\hline \multirow[t]{5}{*}{ Stage } & pTa & $78(90)$ & $40(71)$ \\
\hline & pTis & & $2(4)$ \\
\hline & pT1 & $2(2)$ & 7 (12) \\
\hline & pT2-4 & $5(6)$ & $4(6)$ \\
\hline & pTx & $2(2)$ & $3(7)$ \\
\hline \multirow[t]{4}{*}{ Grade } & G1 & 25 (29) & $23(35)$ \\
\hline & G2 & $52(60)$ & $13(23)$ \\
\hline & G3 & $8(9)$ & $19(32)$ \\
\hline & Gx & $2(2)$ & $1(10)$ \\
\hline Recurrence-free & $\begin{array}{l}\text { Patients, } \\
\text { no./urine } \\
\text { samples, no. }\end{array}$ & $65 / 232$ & $20 / 20$ \\
\hline
\end{tabular}

NMIBC $=$ non-muscle-invasive bladder cancer.

Relapsing tumors include both recurrences and progression detected in patients with initial diagnosis of NMIBC. histologically proven tumor. Table 2 shows the characteristics of patients included in the urine study.

All patients provided written informed consent. The ethics committees of the participating institutions approved all studies. Samples from the Netherlands were obtained according to the Federation of Dutch Medical Scientific Societies' Code for Proper Secondary Use of Human Tissues in the Netherlands (http://www.federa.org/).

\subsection{Cell lines}

UBC cell lines (Supplemental Table 1) were cultured under standard conditions and used for DNA extraction when at 70-80\% confluence. All cultures were Mycoplasma free; their identity was tested by analysis of known genetic mutations.

\subsection{DNA isolation and mutation analyses}

Tumor cell-containing regions were macrodissected from frozen blocks or FFPE sections after examination of hematoxylin and eosin-stained sections. For MIBC cases, DNA was isolated from tumor obtained at the time of cystectomy or, in case of pT0, of incident transurethral resection. Tumor DNA and leukocyte DNA were extracted using the Qiagen kit for blood and tissue, according to manufacturer's instructions (Qiagen $\mathrm{GmbH}$, Hilden, Germany). Sanger sequencing of polymerase chain reaction (PCR) products was used to identify all possible mutations in the first set of patients (Fig. 1A). A 163-base pair (bp) PCR product encompassing the proximal TERT promoter was amplified (primers: CAGCGCTGCCTGAAACTC [forward] and GTCCTGCCCCTCACCTT [reverse]); for some samples, a broader 488-bp region of the promoter was amplified (primers: AGCACCTCGCGGTAGTGG [forward], GGCCGATTCGACCTCTCT [reverse]). 

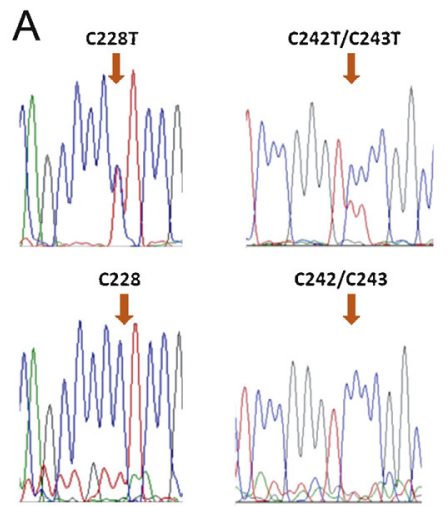

C242/C243

$\downarrow$
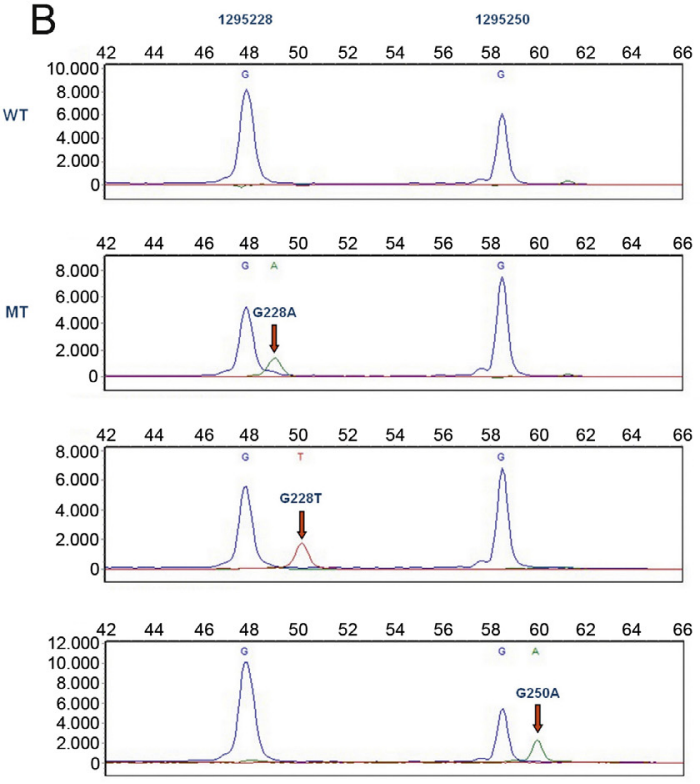
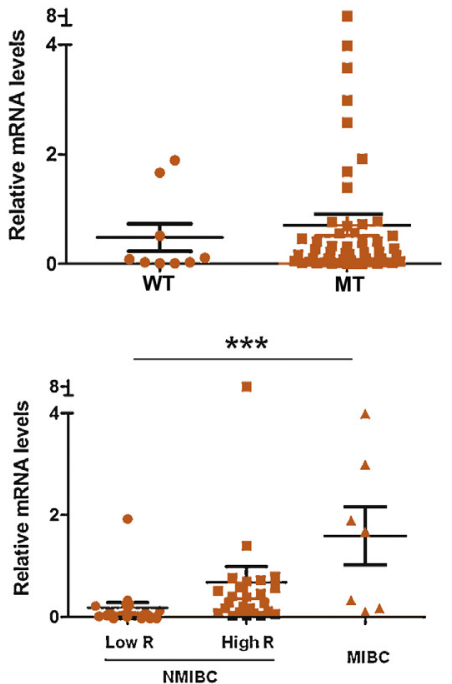

Fig. 1 - Somatic telomerase reverse transcriptase (TERT) promoter mutations in urothelial bladder cancer (UBC). (A) The C228T and C242T/C243T mutations are detected in tumor DNA but not in leukocyte DNA (Sanger sequencing). (B) Mutation assay for the indicated nucleotides in the promoter of the TERT gene. Upper panel: Control sample with wild-type peaks for positions C228T and C250T (from left to right). The lower panels show mutant samples and the nature of the mutation has been indicated. Note that the assay detects the mutations from the complementary strand, hence the mutations are indicated as G228A, G228T, and G250A. (C) Distribution of TERT promoter mutations in UBC according to $T$ and G. Tumors are categorized in three groups: low-risk non-muscleinvasive bladder cancer (NMIBC) (TaG1/G2) (Low R), high-risk NMIBC (TaG3, T1G2/G3) (High R), and MIBC (>T2) for Spain (Sanger sequencing) and the Netherlands (SNaPshot; Applied Biosystems, Foster City, CA, USA). Absolute numbers of cases are given for each mutation and group. (D) TERT messenger RNA levels in bladder tumors assessed by reverse transcriptase-quantitative polymerase chain reaction $(n=60)$; results show the comparison of wild type versus mutant tumors and the comparison of the three tumor groups according to $\mathrm{T}$ and $\mathrm{G} .{ }^{* * *} p \leq 0.001$. WT $=$ wild type; $\mathrm{MT}=$ mutant type.

PCR conditions can be provided on request to the authors. FGFR3 mutation assays were performed as described [8].

For the TERT mutational analysis in the second patient group, we designed a SNaPshot assay (Invitrogen Corp, Carlsbad, CA, USA) through a PCR (155 bp) covering the two most frequent mutations identified (primers: AGCGCTGCCTGAAACTCG [forward], CCCTTCACCTTCCAGCTC [reverse]), similar to the previously designed FGFR3 mutation assay [8]. The PCR was followed by a single-nucleotide extension using probes annealing to the amplicon adjacent to the mutation site. Probe sequences were: T $_{23}$ GGCTGGGAGGGCCCGGA for mutation 1,295,228 (C228T/A) and $\mathrm{T}_{39}$ CTGGGCCGGGGACCCGG for 1,295,250 (C250T) at $1 \mu \mathrm{M}$ and $1.5 \mu \mathrm{M}$, respectively (ABI PRISM SNaPshot Multiplex Kit; Applied Biosystems, Foster City, CA, USA). The products were analyzed (ABI PRISM 3100 Genetic Analyzer; Applied Biosystems, Foster City, CA, USA) with the label indicating the presence or absence of a mutation. Genescan Analysis Software v.3.7 (Applied Biosystems, Foster City, CA, USA) was used for data analysis (Fig. 1B).
For urine DNA extraction and mutation analysis, cell pellets were washed twice with phosphate-buffered saline, after thawing, by centrifugation for $5 \mathrm{~min}$ at $6000 \mathrm{rpm}$ (3000 g). DNA was extracted using the QiAamp Mini and Blood mini kit (Qiagen NV, Venlo, Netherlands) according to the manufacturer's protocol. TERT mutational analysis was performed using the SNaPshot assay.

Reverse transcriptase-quantitative PCR (RT-qPCR) was used to assess TERT RNA expression (see Supplement).

\subsection{Statistical and bioinformatics analyses}

The Predictive Analytics Software v.19 (IBM Corp., Armonk, NY, USA) was used. Statistical differences were considered significant if $p<0.05$. The chi-square and Fisher exact tests were used to determine the relationships between different variables. Statistical differences between groups were assessed using a nonparametric Wilcoxon test, as appropriate. The logrank test was used for survival analysis. In the NMIBC group, all outcomes 
other than progression appear as censored in the Kaplan-Meier curve. Bioinformatics analysis of the TERT promoter sequence is detailed in the Supplement.

\section{Results}

\subsection{TERT promoter mutations in bladder cancer}

We first analyzed the occurrence of mutations in UBC cell lines and tumors using Sanger sequencing of PCR products. Of 32 lines analyzed, 4 were wild type and 28 (87\%) harbored the C228T $(n=25)$ or C250T $(n=3)$ mutations (Supplemental Table 1 ). The somatic nature of these mutations could not be evaluated due to lack of germline DNA.

Among the 111 UBCs from Spain, covering the full spectrum of the localized disease, 78 were mutated (70\%) (Table 1$)$. The most common mutation was C228T $(n=65)$, followed by C250T $(n=10)$; two additional rare mutations were C242T/C243T $(n=2)$ and C228A $(n=1)$. All mutations were mutually exclusive; their somatic nature was confirmed in 10 cases (Fig. 1A). The C250T mutation was significantly less common in UBC than in melanoma $(p=0.00003)$; the $\mathrm{C} 228 \mathrm{~T} / \mathrm{C} 229 \mathrm{~T}$ mutation reported in melanoma was not found in UBC. TERT mutations were not associated with age, sex, or smoking. The frequency of TERT mutations was similar in low-risk NMIBC (TaG1 and TaG2; 33 of 45 [73\%]), high-risk NMIBC (TaG3, Tis, T1G2, T1G3; 34 of $46[74 \%]$ ), and in MIBC (10 of 19 [53\%]) $(p=0.192)$ (Fig. 1C) and in newly diagnosed versus recurrent tumors ( $p=0.716)$. TERT mutations were significantly more frequent among FGFR3 mutant tumors (43 of 49 vs 29 of $54 ; p=0.0002$ ).

We analyzed the two most frequent TERT mutations in an independent set of tumors from the Netherlands using a specifically designed SNaPshot assay; mutations were found to be mutually exclusive. A high mutation rate was confirmed in both NMIBC (147 of 184 [80\%]) and MIBC (136 of 173 [79\%]) (Fig. 1C). To assess heterogeneity, we analyzed TERT mutations using the SNaPshot assay in multiple (median: 4; range: 2-5) regions of nine NMIBCs. We observed general consistency among the regions, suggesting a low level of intraindividual heterogeneity (Supplemental Table 2). These findings place TERT mutations as the most common genetic alteration in UBC [6,7] and as an early event in urothelial carcinogenesis.

TERT mutations have been proposed to generate novel transcription factor binding sites leading to increased TERT expression [12,13]. Mining the DNA-binding factor database JASPAR, we performed a comparative analysis of the wild-type and mutant sequences and found that the C228A mutation newly reported in this paper was also associated with an increased probability of binding to ELK1 (a member of the ETS oncogene family) and v-ets avian erythroblastosis virus E26 oncogene homolog 1 (ETS1), as the previously reported mutations. The analysis revealed additional transcription factors that could contribute to TERT overexpression as well some that showed mutation selectivity, such as v-rel avian reticuloendotheliosis viral oncogene homolog (REL) for C242T/C243T or GATA bind protein 2 (GATA2) for C228A (Supplemental Table 3). To assess the relationship between TERT mutations and mRNA levels, we analyzed 60 primary UBC samples using RT-qPCR. TERT mRNA levels were higher in TERT mutant tumors, but the difference was not statistically significant (Wilcoxon test $p=0.3$ ). TERT mRNA levels were significantly higher in high-risk NMIBC and in MIBC than in low-risk NMIBC (Wilcoxon test $p=0.001$ ) (Fig. 1D).

\subsection{TERT mutations and patient outcome}

The set of tumors from the Netherlands was used to assess tumor prognostic value. Of 184 patients with NMIBC, 90 (49\%) experienced progression to MIBC (Table 1). There was no relationship between progression-free survival and the presence of mutations (log-rank test $p=0.984$ ) (Supplemental Fig. 1). In the group of 173 patients with MIBC, 78 (45\%) patients had local or distant progression and TERT mutation status was not associated with progression-free survival (log-rank test $p=0.160)$. Furthermore, 101 (58\%) patients died during follow-up, including 65 (37\%) who died of bladder cancer (Table 1). Overall- and cancer-specific survival were not related to TERT mutations (log-rank tests $p=0.987$ and $p=0.834$ ) (Supplemental Fig. 1). Among patients with MIBC, 25 received adjuvant chemotherapy with curative intent; TERT mutations were not predictive for survival in this subgroup or the group without chemotherapy.

Table 3 - Sensitivity and specificity of urine diagnostic assays

\begin{tabular}{|c|c|c|c|c|c|}
\hline & Variable & $\begin{array}{c}\text { The Netherlands, } \\
\text { no./total (\%) }\end{array}$ & $\begin{array}{l}\text { Spain, } \\
\text { no./total (\%) }\end{array}$ & $\begin{array}{l}\text { Netherlands and } \\
\text { Spain, no./total (\%) }\end{array}$ & $\begin{array}{l}\text { Netherlands and Spain, } \\
\qquad p \text { value }\end{array}$ \\
\hline \multirow[t]{3}{*}{ Primary tumors, sensitivity } & FGFR3 & $17 / 42(40)$ & $29 / 86(34)$ & $46 / 128(36)$ & - \\
\hline & TERT & $21 / 37(57)$ & $52 / 81(64)$ & $73 / 118(62)$ & FGFR3 vs TERT, $p<0.0001$ \\
\hline & TERT + FGFR3 & $20 / 28(71)$ & $53 / 77$ (69) & $73 / 105(70)$ & TERT vs TERT + FGFR3, $p=0.23$ \\
\hline \multirow[t]{3}{*}{ Recurrent tumors, sensitivity } & FGFR3 & $15 / 68(22)$ & $9 / 56(16)$ & $24 / 124(19)$ & - \\
\hline & TERT & $18 / 63(29)$ & $30 / 50(60)$ & 48/113 (42) & FGFR3 vs TERT, $p=0.0001$ \\
\hline & TERT + FGFR3 & $21 / 53(40)$ & $31 / 50(62)$ & $52 / 103(50)$ & TERT vs TERT + FGFR $3, p=0.238$ \\
\hline \multirow{3}{*}{$\begin{array}{l}\text { Recurrence-free FU urine } \\
\text { samples, specificity }\end{array}$} & FGFR3 & $208 / 232(90)$ & $19 / 20(95)$ & $227 / 252(90)$ & - \\
\hline & TERT & $149 / 200(75)$ & $11 / 18(61)$ & $160 / 218(73)$ & FGFR3 vs TERT, $p<0.0001$ \\
\hline & $T E R T+F G F R 3$ & $131 / 183(72)$ & $11 / 18(61)$ & $142 / 201(71)$ & TERT vs TERT + FGFR3, $p=0.529$ \\
\hline
\end{tabular}

FGFR3 = fibroblast growth factor receptor 3; FU = follow-up; TERT = telomerase reverse transcriptase. 


\subsection{TERT mutation detection in exfoliated cells in urine}

Patients with NMIBC require continued follow-up due to frequent tumor recurrences. We assessed whether TERT mutations can be detected in exfoliated tumor cells in voided urine and compared the findings with FGFR3 mutations (Table 3). Of 39 urine samples from patients with hematuria and in whom no bladder tumor was visible at cystoscopy ( 17 of 39 [44\%] female; median age: $54 \mathrm{yr}$ ), 4 were positive for TERT mutations, resulting in a specificity of $90 \%$. None of these four patients developed a UBC or another tumor in the subsequent $>3.5 \mathrm{yr}$. In urine samples obtained before resection of a primary bladder tumor, the sensitivity of incident tumor detection was 36\% (46 of 128) for FGFR3, $62 \%$ ( 73 of 118 ) for TERT, and 70\% (73 of 105) for TERT and FGFR3 combined (FGFR3 vs TERT, $p<0.0001$ ). We then tested 395 urine samples prospectively collected from 194 patients under surveillance after a primary NMIBC diagnosis. Sensitivity of detection of relapsing (recurrence or progression) UBC developing after a primary NMIBC was 19\% (24 of 124 ) for FGFR3, 42\% (48 of 113) for TERT, and 50\% (52 of 103) for both combined (FGFR3 vS TERT, $p=0.0001$ ). In the recurrence-free urine samples, a TERT mutation was detected in 58 of 218 cases, corresponding to a specificity of $73 \%$, whereas the specificity for FGFR3 mutation was $90 \%$ (FGFR3 vs TERT, $p<0.0001$ ).

\section{Discussion}

TERT promoter hotspot mutations constitute a new type of somatic genetic alterations in cancer. A recent survey in a wide range of tumors indicated a selective mutational pattern associated with tumor histology and tissue of origin [14]. It has been proposed that mutations occur more commonly in tumors derived from tissues with low proliferation rates [12-14]. Based on the analysis of 21 UBCs, a high mutation rate was proposed, but there was no clinical or pathologic information on these patients.

In this paper, we provide definitive evidence on the occurrence of TERT promoter mutations in UBC. First, we show a high mutation rate (70\%), which was replicated in an independent series (79\%) using different assays, thus making TERT the most frequently mutated gene in UBC. Second, we show a similar mutation frequency across stages and grades, strongly suggesting that TERT mutations participate in the two major genetic pathways involved in UBC. Third, we show a distinct mutational spectrum in comparison with melanoma with a dominance of the C228T mutation. Finally, we show a lack of association with progression in NMIBC or with the development of local or distant metastases in MIBC.

The high frequency of hotspot mutations renders TERT a very attractive target for diagnosis of bladder tumors, both primary and recurrent, using body fluids such as urine or blood. We show that tumor cell detection in urine is feasible, with a sensitivity of $62 \%$ at initial diagnosis and $42 \%$ at recurrence. For urine diagnostics during surveillance, the patients were not stratified for mutation status of the primary tumor; we expect that when this is performed, sensitivity of detection of recurrent UBC will increase. Accordingly, TERT mutation was assayed concomitantly in tumor tissue and urine in 31 cases: TERT was concordant in 26 cases (19 mutated, 7 wild type), one tumor harbored a C242T mutation not assessable by the SNaPshot assay, and four mutations were detected in urine but not in tumor samples. We show that TERT mutation detection has a higher sensitivity than FGFR3 mutation for tumor cell detection in urine, and assays of both TERT and FGFR3 mutations might have improved sensitivity, though the difference was not statistically significant in our study. By contrast, TERT mutations may have a lower specificity than FGFR3 mutations, as four mutations were detected in 39 urine samples from patients without cancer and in 58 of 218 of patients in clinical remission. In this group, we cannot distinguish reduced specificity from higher leadtime bias in detection of tumor relapse. Larger, welldesigned, prospective studies are required to establish the clinical usefulness of mutation detection in urine. As previous reports have proposed TERT expression or activity assessment in urine as a tool to detect recurrence, it will be of high interest to compare TERT mutation with them and with other candidate biomarkers in future studies $[17,18]$.

Several observations support the notion that TERT somatic mutations are an early event in urothelial carcinogenesis, including their occurrence in a small fraction of subjects without UBC, their presence in tumors of both the papillary and invasive pathways, and the low level of intraindividual heterogeneity when multiple tumor regions were analyzed. UBC cell lines are also very frequently mutated, and we noticed that most of the mutant ones lacked the wild-type allele. These observations also suggest that mutant cells may display a growth advantage over wild-type cells.

The mechanisms through which TERT promoter mutations contribute to UBC and other tumors remain to be determined. One possibility is that the acquisition of new, putative transcription binding sites leads to altered regulation of gene expression, as previously proposed, and also suggested by our bioinformatics analysis using the DNA-binding factor database JASPAR. However, we failed to find a significant association between TERT mutation and expression. This could be explained by alternative mechanisms of TERT upregulation in wild-type tumors, also leading to TERT overexpression, or by the participation of TERT mutations in additional biologic processes.

Given its reverse transcriptase activity, telomerase is a potential therapeutic target. Recent developments suggest that azidothymidine (AZT), an inhibitor of HIV reverse transcriptase, also inhibits telomerase. Hence, it is conceivable that AZT or other telomerase inhibitors may suppress the growth of UBC overexpressing the enzyme [19].

One of the limitations of our work is the retrospective nature of the cohorts used for the outcome analysis, even though our results do not make positive claims. Another is the lack of comparison for urine-based diagnostic assays between TERT mutation, cytology, and other biomarkers. However, the high frequency of these mutations in urine reported herein provides evidence for future prospective and comparative studies. Overall, the novelty of TERT mutation in 
UBC is strengthened by the independent analysis that we performed in two different centers.

\section{Conclusions}

Mutations in the promoter of the TERT gene are the most frequent somatic mutations in tumors of the urinary bladder. Mutations occur with similar frequency, regardless of stage or grade, and are not associated with clinical outcome. TERT mutation assays may be used for tumor cell detection in urine and TERT may be a therapeutic target.

Author contributions: Francisco X. Real had full access to all the data in the study and takes responsibility for the integrity of the data and the accuracy of the data analysis.

Study concept and design: Allory, Malats, Zwarthoff, Real.

Acquisition of data: Allory, Beukers, Sagrera, Flández, Miriam Marqués, Mirari Márquez, van der Keur, Dyrskjot, Lurkin, Vermeij, Carrato, Lloreta, Lorente, Carrillo-de Santa Pau, Masius, Kogevinas, Steyerberg, van Tilborg, Abas, Orntoft, Zuiverloon.

Analysis and interpretation of data: Allory, Beukers, Sagrera, Flández, Carrillo, Malats, Zwarthoff, Real.

Drafting of the manuscript: Allory, Zwarthoff, Real.

Critical revision of the manuscript for important intellectual content: Malats. Statistical analysis: Malats.

Obtaining funding: Malats, Zwarthoff, Real.

Administrative, technical, or material support: None.

Supervision: Real, Zwarthoff.

Other (specify): None.

Financial disclosures: Francisco X. Real certifies that all conflicts of interest, including specific financial interests and relationships and affiliations relevant to the subject matter or materials discussed in the manuscript (eg, employment/affiliation, grants or funding, consultancies, honoraria, stock ownership or options, expert testimony, royalties, or patents filed, received, or pending), are the following: None.

Funding/Support and role of the sponsor: This work was supported, in part, by grants from Ministerio de Economía y Competitividad, Madrid (grants Consolíder ONCOBIO and SAF2011-15934-E), Instituto de Salud Carlos III (grants G03/174, 00/0745, PI051436, PI061614, G03/174, and Red Temática de Investigación Cooperativa en Cáncer (RTICC), Asociación Española Contra el Cáncer, the Dutch Cancer Society (EMCR 2007-3863) and by a European Community Seventh Framework program FP7/20072013, no. 201663. Sponsors contributed to data collection and preparation.

Acknowledgment statement: The authors wish to thank the pathologists and urologists from the Haga, Reinier de Graaf, Amphia, and St. Fransiscus hospitals for making available the tumor tissue blocks and patient data, and F. Algaba, M. Calle, I. Flores, X. Langa, T. Lobato, P.U. Malmström, X. Mayol, M. Méndez-Pertuz, and A.C. Picornell for valuable contributions. The samples of the pTa tumors were obtained with help of the Dutch Pathology National database PALGA.

\section{Appendix A. Supplementary data}

Supplementary data associated with this article can be found, in the online version, at http://dx.doi.org/10.1016/ j.eururo.2013.08.052.

\section{References}

[1] Resnick MJ, Bassett JC, Clark PE. Management of superficial and muscle-invasive urothelial cancers of the bladder. Curr Opin Oncol 2013;25:281-8.

[2] Babjuk M, Oosterlinck W, Sylvester R, et al. EAU guidelines on nonmuscle-invasive urothelial carcinoma of the bladder, the 2011 update. Eur Urol 2011;59:997-1008.

[3] Stenzl A, Cowan NC, De Santis M, et al. Treatment of muscleinvasive and metastatic bladder cancer: update of the EAU guidelines. Eur Urol 2011;59:1009-18.

[4] Cappellen D, De Oliveira C, Ricol D, et al. Frequent activating mutations of FGFR3 in human bladder and cervix carcinomas. Nat Genet 1999;23:18-20.

[5] Billerey C, Chopin D, Aubriot-Lorton MH, et al. Frequent FGFR3 mutations in papillary non-invasive bladder (pTa) tumors. Am J Pathol 2001;158:1955-9.

[6] Luis NM, López-Knowles E, Real FX. Molecular biology of bladder cancer. Clin Transl Oncol 2007;9:5-12.

[7] Kompier LC, Lurkin I, van der Aa MN, van Rhijn BW, van der Kwast TH, Zwarthoff EC. FGFR3, HRAS, KRAS, NRAS and PIK3CA mutations in bladder cancer and their potential as biomarkers for surveillance and therapy. PloS One 2010;5:e13821.

[8] Van Oers JM, Lurkin I, van Exsel AJ, et al. A simple and fast method for the simultaneous detection of nine fibroblast growth factor receptor 3 mutations in bladder cancer and voided urine. Clin Cancer Res 2005;11:7743-8.

[9] Zuiverloon TC, van der Aa MN, van der Kwast TH, et al. Fibroblast growth factor receptor 3 mutation analysis on voided urine for surveillance of patients with low-grade non-muscle-invasive bladder cancer. Clin Cancer Res 2010;16:3011-8.

[10] Shay JW, Wright WE. Role of telomeres and telomerase in cancer. Semin Cancer Biol 2011;21:349-53.

[11] Günes C, Rudolph KL. The role of telomeres in stem cells and cancer. Cell 2013;152:390-3.

[12] Huang FW, Hodis E, Xu MJ, Kryukov GV, Chin L, Garraway LA. Highly recurrent TERT promoter mutations in human melanoma. Science 2013;339:957-9.

[13] Horn S, Figl A, Rachakonda PS, et al. TERT promoter mutations in familial and sporadic melanoma. Science 2013;339:959-61.

[14] Killela PJ, Reitman ZJ, Jiao Y, et al. TERT promoter mutations occur frequently in gliomas and a subset of tumors derived from cells with low rates of self-renewal. Proc Natl Acad Sci U S A 2013;110: 6021-6.

[15] García-Closas M, Malats N, Silverman D, et al. NAT2 slow acetylation, GSTM1 null genotype, and risk of bladder cancer: results from the Spanish Bladder Cancer Study and meta-analyses. Lancet 2005;366:649-59.

[16] Hernández S, López-Knowles E, Lloreta J, et al. Prospective study of FGFR3 mutations as a prognostic factor in nonmuscle invasive urothelial bladder carcinomas. J Clin Oncol 2006;24:3664-71.

[17] Sanchini MA, Gunelli R, Nanni O, et al. Relevance of urine telomerase in the diagnosis of bladder cancer. JAMA 2005;294: 2052-6

[18] Tilki D, Burger M, Dalbagni G, et al. Urine markers for detection and surveillance of non-muscle-invasive bladder cancer. Eur Urol 2011;60:484-92.

[19] Gomez DE, Armando RG, Alonso DF. AZT as a telomerase inhibitor. Front Oncol 2012;2:113. 